\title{
ON THE REGISTRATION SYSTEM OF THE AIST-PROJECT
}

\author{
M.S.CHUBEY, V.S.PASHKOV, I.M.KOPYLOV, T.R.KIRIAN AND \\ V.V.NICKIFOROV \\ Pulkovo Observatory, 196140, St.-Petersburg, Russia \\ e-mail: mchubey@gaoran.spb.su
}

AND

S.V.MARKELOV AND V.P.RYADCHENKO

Special Astrophys. Observatory,

Nizhnij Arkhyz, Karachai-Circassian Republic, Russia, 357147

e-mail:markel@sao.stavropol.su

\begin{abstract}
The researches on the AIST-project are directed to the design of the focal plane assembly. The concept of the chip-mosaic and numeric simulation of the two-pixels mode of the signal sampling are presented.
\end{abstract}

\section{Introduction}

The work on the AIST-project was carried out in accordance with the concept described by Chubey et al. (1993). More recently the main researches have been devoted to the registration system on CCD chips.

The increase of the micrometer efficiency by 2-3 orders in the number of objects that can be registered and the increase of the astrometric and photometric accuracy because of the longer registration time - all this has changed the scope of task for the forthcoming second generation space astronomy experiment. Its scientific content evidently will be a close analogy with the "Carte du Ciel" with natural development towards electronic tools of registration and data processing. The data base which is planned to be gathered in the form of astrometric and photometric catalogues, will be of interest as the source material for fundamental investigations in astronomy. This opinion is supported by the AIST team being operating on the international cooperation. The goal of this paper is to present short information about the current stage of technical design of the project, beyond any crit- 
icism of the concurrent projects, and mainly concerning the problem of the registration system.

\section{Particular decisions in the project}

Two telescopes on board in a stable arrangement allow to use three fields on the scanning great circle, two of which $R_{1}$ and $S_{1}$, Fig. 1 , are folded in the focal plane $F_{1}$ by means of a beam combiner; the third is a full aperture single optical channel to the second focal plane $F_{2}$. This variant was discussed and described earlier (Høg and Chubey 1991). The advantages of this variant are:

- the ability to use the hardware and algorithmic separation of the folded fields $R_{1}, S_{1}$ with the simple subsequent separation of the frames $R_{1}$ and $S_{1}$ from their folded image $R_{1}+S_{1}$, aided by the focal assembly $F_{2}$;

- photometric gain is evident: the round and twice as bright optical image of every object in the field $F_{2}$ doubles the "signal-to-noise" ratio;

- the choice of the angle between $R_{1}$ and $R_{2}$ is $\gamma_{1}=180^{\circ}$, implying absolutely symmetrical paths of the centers of areas $R_{1}$ and $R_{2}$ on the scanning motion.

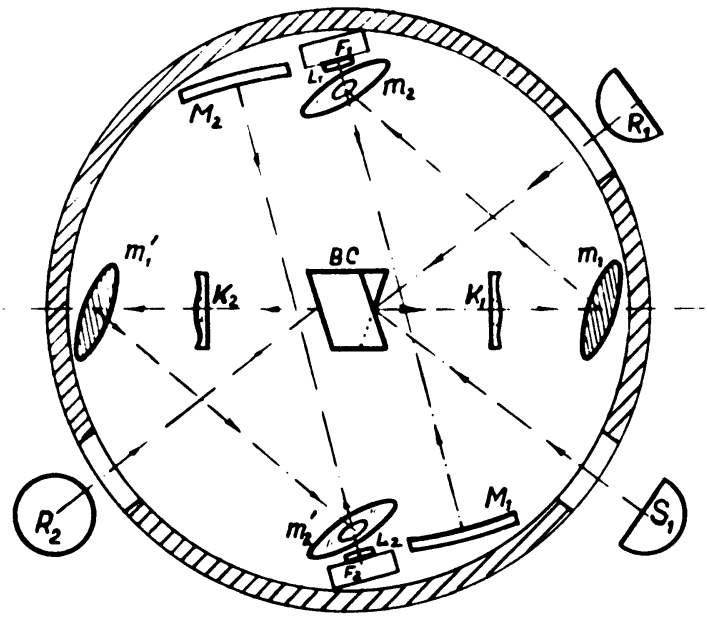

Figure 1. Option with a full aperture entrance pupil. Both focal plane assemblies $F_{1}, F_{2}$ include the field lenses $L_{1}, L_{2}$.

The definition of the attitude angles under the chosen dynamics of the space craft will be processed more precisely because of the near-rectangular distribution of the scanned directions: the base angle between $R_{1}, S_{1}$ is $\gamma_{2}=74^{\circ}$ in the discussed variant. 
The main disadvantage of the double-telescope instrument is the rise of the information flow.

\section{Micrometer based on CCD chips}

The detailed research and design of the micrometer requires long time and great efforts. Only a short conclusion from our analysis of the problem is presented.

The use of micro channel plates chip for the micrometer is not supposed in our analysis because of the low $\mathrm{QE}$, more narrow spectral band and the high level of noise on the cascade amplifier: the puls amplitude spectrum has the Farri's distribution (negative exponent), with the dispersion of the amplification coefficient equal to the square of this coefficient. This can not be improved significantly by hardware. The most important disadvantages are the loss of astrometric accuracy and the narrow dynamical range.

The CCD in fact is a television sensor, working in the super-smallframe regime with large possibilities to suppress the noises. Manufactured by ELECTRON Company (Russia) chips with $16 \times 16 \mu m$ pixels have a wellcapacity up to $130000 e^{-}$with a read-out noise of $5 e^{-}$, covering a dynamic range of 26000 , i.e. near to 11 magnitudes. The world level is somewhat better. We assume that the time delay and integration, (TDI) principle, or variant of it, must be the basic mode of operation.

The integration mode suggested by Høg and Lindegren (1993), based on the TDI, allows to analyze the photometric profile of the diffraction image of star. The splitting of the image in $N$ parts applied to CCD could ensure a higher accuracy, but the large number of transfers demands a charge transfer efficiency of nearly 0.999998 and leads to a decrease of the energy and restriction of the photometric possibilities. The accuracy estimation of the form modified by Yershov (1993), shows that the optimal number of splits for the AIST optics is $N=3$.

An extended simulation was made for the TDI subclass (Pashkov \& Chubey 1993) using two-pixel integration method. The exposure $T=8.8 \mathrm{~ms}$ followed by a synchronous shift forward were used when the star image crosses each pixel. A polychromatic model of the Point-Spread Function and the discriminating function were calculated for the high level noise condition. In Fig. 2 the plots of summarized astrometric accuracies are shown. The Monte-Carlo simulation with 20 tests and 512 pixels path in the array column were used. Two levels of the readout noises $5 e^{-}, 10 e^{-}$were used in the model and two Analog Digital Convertors (ADC) 8 and 12-bit encoding, for red and blue spectral bands, for three variants of chips $(128,256$ and 512 pixels in column) - all these in the range of $8-18$ magnitudes. 


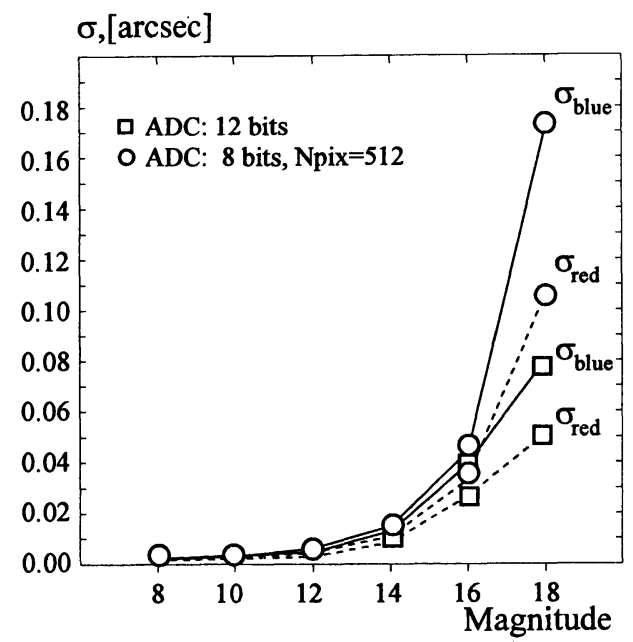

Figure 2. The one-array-readout error $\sigma$ (mas) vs star magnitude.

\section{The working version of a CCD-camera design}

To perform the 7-colour photometry a larger number of CCDs must be used compared with the Roemer mission (Høg 1993). Two focal assemblies will be used. In each the CCD sensor must be a mosaic of 3 or $4 \times 10$ chips with the number of elements $512 \times 512$. Each CCD-array has a read-out system with virtual transfer of the frame and division of the horizontal register into two equal parts, each of which is read out on different sides. The small variations of the transfer speed, the usage of matrixes with nonperiodical structure and other methods from the TDI regime - all these are under careful testing for optimal final choice.

\section{References}

Chubey, M.S. et al., (1993), Aist Project: Scientific and Technological Foundations, Developments in Astrometry and Their Impact on Astrophysics and Geodynamics, I.I.Mueller \& B.Kolaczek (eds.), IAU Symp.156, pp. 415-420.

Høg, E., Chubey, M.S., (1991), Proposal for the Second Hipparcos, Intern.Symp. "Etalon91 ", Moscow, unpublished.

$\mathrm{H} \varnothing \mathrm{g}$, E., (1993), Astrometry and Photometry of 400 Million Stars Brighter than $18 \mathrm{Mag}$, Developments in Astrometry and Their Impact on Astrophysics and Geodynamics, I.I.Mueller \& B.Kolaczek (eds.), IAU Symp.156, pp. 37-45.

$\mathrm{H} \varnothing \mathrm{g}, \mathrm{E}$., Lindegren, L., (1993), A CCD Modulation Detector for a Second HIPPARCOS Mission, ibid, pp. 31-36.

Pashkov, V.S., Chubey, M.S., (1993), On the Accuracy Estimation in the AIST-Project, Proceed. of the Russian Astrometric Conf. held in St.-Petersburg, Oct. 4-8, 1993, in press.

Yershov, V., (1993), A Focal CCD Micrometer for the Astrometric Satellite, Second Intern. Workshop on Posit. Astronomy and Celest. Mech., ed. A.Lopez Garcia, Valencia Univ., pp. 307-312. 